\title{
Nonlinear Bi-Integrable Couplings of Multicomponent Guo Hierarchy with Self-Consistent Sources
}

\author{
Hongwei Yang, ${ }^{1}$ Huanhe Dong, ${ }^{1}$ Baoshu Yin, ${ }^{2,3}$ and Zhenyu Liu ${ }^{4}$ \\ ${ }^{1}$ Information School, Shandong University of Science and Technology, Qingdao 266590, China \\ ${ }^{2}$ Institute of Oceanology, China Academy of Sciences, Qingdao 266071, China \\ ${ }^{3}$ Key Laboratory of Ocean Circulation and Wave, Chinese Academy of Sciences, Qingdao 266071, China \\ ${ }^{4}$ Department of Mathematics, Zaozhuang College, Zaozhuang 277160, China
}

Correspondence should be addressed to Baoshu Yin, baoshuyin@126.com

Received 4 October 2012; Accepted 5 December 2012

Academic Editor: D. E. Pelinovsky

Copyright (C) 2012 Hongwei Yang et al. This is an open access article distributed under the Creative Commons Attribution License, which permits unrestricted use, distribution, and reproduction in any medium, provided the original work is properly cited.

\begin{abstract}
Based on a well-known Lie algebra, the multicomponent Guo hierarchy with self-consistent sources is proposed. With the help of a set of non-semisimple Lie algebra, the nonlinear biintegrable couplings of the multicomponent Guo hierarchy with self-consistent sources are obtained. It enriches the content of the integrable couplings of hierarchies with self-consistent sources. Finally, the Hamiltonian structures are worked out by employing the variational identity.
\end{abstract}

\section{Introduction}

Since the notion of integrable couplings was proposed in view of Virasoro symmetric algebras $[1,2]$ and the soliton theory, considerable research has been reported on the integrable couplings and associated properties of some known interesting integrable hierarchies, such as the AKNS hierarchy, the KN hierarchy, and the Burger hierarchy [3-6]. In order to produce multicomponent integrable systems, a type of multicomponent loop algebra $\tilde{G}_{M}$ was structured, and it followed that some multicomponent integrable systems were given and Hamiltonian structures of multicomponent systems were constructed through the component-trace identity in [7-13] and a general structure between Lie algebras and integrable coupling was given in $[14,15]$. Here it is necessary to point out that the abovementioned integrable couplings are linear for the supplementary variable, so they are called linear integrable couplings. Recently, Ma proposed the notion of nonlinear integrable couplings and gave the general scheme to construct the nonlinear integrable couplings of 
hierarchies [16]. In $[17,18]$, based on the general scheme of constructing nonlinear integrable couplings, Zhang introduced some new explicit Lie algebras and obtained the nonlinear integrable couplings of the GJ hierarchy, the Yang hierarchy, and the CBB hierarchy. In [19], Ma employed a class of non-semisimple matrix loop algebras to generate bi-integrable couplings of soliton equation from the zero curvature equations.

On the other hand, with the development of soliton theory, people began to focus on the soliton equation with self-consistent sources [20,21]. The soliton equations with self-consistent sources have important physical meaning and are often used to express interactions between different solitary waves and are relevant to some problems of hydrodynamics, solid state physics, plasma physics, and so on [22, 23]. In [24-26], Yu and Xia discussed the integrable couplings of hierarchies with self-consistent sources and obtained a series of integrable hierarchies with self-consistent sources and their couplings systems as well as Hamiltonian structures. In these papers, the integrable couplings are linear for supplementary variables.

As mentioned above, we will find that there are very few researches on the nonlinear integrable couplings of hierarchies with self-consistent sources available up till now, especially on the nonlinear bi-integrable couplings of multicomponent hierarchy. In our work, we will derive the nonlinear bi-integrable couplings of multicomponent Guo hierarchy with self-consistent sources and their Hamiltonian structures. It will enrich the content of the integrable couplings of hierarchies with self-consistent sources. The plan of the paper is as follows. In Section 2, the multicomponent Guo hierarchy with self-consistent sources is given. By employing the variational identity, the Hamiltonian structure of the above hierarchy is obtained. The notion of bi-integrable couplings is introduced and the nonlinear bi-integrable couplings of the multicomponent Guo hierarchy with self-consistent sources and their Hamiltonian structures are derived in Section 3. Finally, some conclusions are given in Section 4.

\section{Multicomponent Guo Hierarchy with Self-Consistent Sources}

In this section, we recall briefly some basic notions from the theory of the multicomponent integrable system. If $F_{M}=\left\{a=\left(a_{i j}\right)_{M \times 3}=\left(a_{1}, a_{2}, a_{3}\right)\right\}$ denotes a class of matrices where $a_{i}(i=1,2,3)$ is the $i$ th column of the matrix $a, M$ stands for an arbitrary positive integer, then $F_{M}$ is a linear space.

Definition 2.1 (see [7]). Let $\alpha=\left(\alpha_{1}, \alpha_{2}, \ldots, \alpha_{M}\right)^{T}$ and $\beta=\left(\beta_{1}, \beta_{2}, \ldots, \beta_{M}\right)^{T}$, their product is defined as

$$
\alpha * \beta=\beta * \alpha=\left(\alpha_{1} \beta_{1}, \alpha_{2} \beta_{2}, \ldots, \alpha_{M} \beta_{M}\right)^{T}, \quad \frac{\alpha}{\beta}=\left(\frac{\alpha_{1}}{\beta_{1}}, \frac{\alpha_{2}}{\beta_{2}}, \ldots, \frac{\alpha_{M}}{\beta_{M}}\right)^{T} .
$$

Definition 2.2 (see [7]). Set $a=\left(a_{1}, a_{2}, a_{3}\right)$ and $b=\left(b_{1}, b_{2}, b_{3}\right) \in F_{M}$, a commutation operation is defined as

$$
[a, b]=\left(a_{2} * b_{3}-a_{3} * b_{2}, 2\left(a_{1} * b_{2}-a_{2} * b_{1}\right), 2\left(a_{3} * b_{1}-a_{1} * b_{3}\right)\right)^{T}
$$


It is easy to verify that $F_{M}$ is a Lie algebra. Its corresponding loop algebra $\widetilde{F}_{M}$ is

$$
\widetilde{F}_{M}=\left\{a \lambda^{n}, a \in F_{M}, n=0, \pm 1, \pm 2, \ldots\right\},
$$

equipped with a commutation operation as follows:

$$
\left[a \lambda^{m}, b \lambda^{n}\right]=[a, b] \lambda^{m+n}, \quad \forall a, b \in F_{M}
$$

Consider the following isospectral problem:

$$
\varphi_{x}=[U, \varphi], \quad \lambda_{t}=0, \quad U=\left(\frac{\lambda I_{M}}{2}, \frac{\left(u_{1}+u_{2}\right)}{2}, \frac{\lambda\left(u_{1}-u_{2}\right)}{2}\right)^{T},
$$

where $u_{1}, u_{2}$ are potentials, $\lambda$ is the spectral parameter, $u_{i}=\left(u_{i 1}, u_{i 2}, \ldots, u_{i M}\right)^{T}, i=1,2$, and $I_{M}=(\underbrace{1,1, \ldots, 1}_{M})^{T}$. By means of constructing a proper time evolution

$$
\varphi_{t}=V \varphi, \quad V=\left(\lambda v_{1}, v_{2}+v_{3}, \lambda\left(v_{2}-v_{3}\right)\right)^{T},
$$

where $v_{k}=\sum_{m=0}^{\infty}\left(v_{k_{1}, m}, v_{k_{2}, m}, \ldots, v_{k_{M}, m}\right)^{T} \lambda^{-m}, k=1,2,3$. Then solving stationary zero curvature equation $V_{x}=[U, V]$, one arrives at the recursion relation as follows:

$$
\begin{aligned}
& v_{1, m x}=u_{2} * v_{2, m}-u_{1} * v_{3, m}, \\
& v_{2, m x}=v_{3, m+1}-u_{2} * v_{1, m+1}, \\
& v_{3, m x}=v_{2, m+1}-u_{1} * v_{1, m+1}, \\
& v_{1,0}=0 I_{M}, \quad v_{2,0}=u_{1}, \quad v_{3,0}=u_{2}, \quad v_{1,1}=\frac{\left(u_{2} * u_{2}-u_{1} * u_{1}\right)}{2}, \\
& v_{2,1}=u_{2, x}+u_{1} * \frac{\left(u_{2} * u_{2}-u_{1} * u_{1}\right)}{2}, \quad v_{3,1}=u_{1, x}+u_{2} * \frac{\left(u_{2} * u_{2}-u_{1} * u_{1}\right)}{2} .
\end{aligned}
$$

For an arbitrary natural number $n$, denoting

$$
V_{+}^{(n)}=\sum_{m=0}^{n}\left(\lambda v_{1, m}, v_{2, m}+v_{3, m}, \lambda\left(v_{2, m}-v_{3, m}\right)\right)^{T} \lambda^{n-m}=\lambda^{n} V-V_{-}^{(n)},
$$

then the stationary zero curvature equation can be written as

$$
-V_{+x}^{(n)}+\left[U, V_{+}^{(n)}\right]=V_{-x}^{(n)}-\left[U, V_{-}^{(n)}\right]
$$


a direct calculation reads

$$
-V_{+x}^{(n)}+\left[U, V_{+}^{(n)}\right]=-\left(0, \frac{\left(v_{2, n x}+v_{3, n x}\right)}{2}, \frac{\lambda\left(v_{2, n x}-v_{3, n x}\right)}{2}\right) .
$$

Then the zero curvature equation

$$
U_{t}-V_{x}^{(n)}+\left[U, V^{(n)}\right]=0
$$

gives the integrable system

$$
U_{t}=\left(\begin{array}{l}
u_{1} \\
u_{2}
\end{array}\right)_{t}=\left(\begin{array}{l}
v_{2, n x} \\
v_{3, n x}
\end{array}\right)=\left(\begin{array}{cc}
\partial & 0 \\
0 & -\partial
\end{array}\right)\left(\begin{array}{c}
v_{2, n} \\
-v_{3, n}
\end{array}\right)=J_{1} L_{1}^{n}\left(\begin{array}{l}
u_{1} \\
u_{2}
\end{array}\right)
$$

where $J_{1}$ is a Hamiltonian operator, and the recurrence operator $L_{1}$ is given from (2.7) by

$$
L_{1}=\left(\begin{array}{cc}
-u_{1} * \partial^{-1} u_{1} * \partial & -\partial-u_{1} * \partial^{-1} u_{2} * \partial \\
-\partial+u_{2} * \partial^{-1} u_{1} * \partial & u_{2} * \partial^{-1} u_{2} * \partial
\end{array}\right)
$$

Accordingly, when $M=1$, the system (2.12) reduces to the Guo hierarchy in [5]; when $M>$ 1 , the system (2.12) is a multicomponent Guo hierarchy. We observe that $J_{1} L_{1}=L_{1}^{*} J_{1}$, the Hamiltonian functions $H_{n}(n \geq 1)$ are involutive with each other, and each $H_{n}$ is a common conserved density of the expression (2.12). Therefore, the multicomponent Guo hierarchy (2.12) is Liouville integrable.

In order to deduce the Hamiltonian structure of (2.12), we rewrite (2.2) as follows:

$$
[a, b]=a^{T} * R_{1}(b)
$$

where

$$
R_{1}(b)=\left(\begin{array}{ccc}
0 & 2 b_{2} & -2 b_{3} \\
b_{3} & -2 b_{1} & 0 \\
-b_{2} & 0 & 2 b_{1}
\end{array}\right)
$$

Solving the matrix equation for the constant matrix $F: R(b) * F=-(R(b) * F)^{T}, F^{T}=F$, presents

$$
F=\left(\begin{array}{ccc}
2 I_{M} & 0 & 0 \\
0 & 0 & I_{M} \\
0 & I_{M} & 0
\end{array}\right)
$$

Then in terms of $F$, define a linear functional:

$$
\{a, b\}=a^{T} * F * b=2 a_{1} * b_{1}+a_{3} * b_{2}+a_{2} * b_{3} .
$$


It is easy to find that $\{a, b\}$ satisfies the variational identity

$$
\frac{\delta}{\delta u}\left\{V, U_{\curlywedge}\right\}=\lambda^{-r} \frac{\partial}{\partial \lambda} \lambda^{r}\left\{V, U_{u}\right\}
$$

By using the above linear functional (2.17), according the variational identity, we have

$$
\frac{\delta}{\delta u}\left(v_{1}+\left(u_{1}-u_{2}\right) * \frac{\left(v_{2}+v_{3}\right)}{2}\right)=\lambda^{-r} \frac{\partial}{\partial \lambda} \lambda^{r}\left(v_{2},-v_{3}\right)^{T} .
$$

Comparing the coefficients of $\lambda^{-n-1}$ yields

$$
\frac{\delta}{\delta u}\left(v_{1, n+1}+\left(u_{1}-u_{2}\right) * \frac{\left(v_{2, n}+v_{3, n}\right)}{2}\right)=(\gamma-n)\left(v_{2, n},-v_{3, n}\right)^{T} .
$$

Taking $n=0$ in the above equation gives $\gamma=0$.

Hence, the Guo hierarchy (2.12) can be written as a Hamiltonian form

$$
U_{t}=\left(\begin{array}{l}
u_{1} \\
u_{2}
\end{array}\right)_{t}=J_{1} L_{1}^{n}\left(\begin{array}{l}
u_{1} \\
u_{2}
\end{array}\right)=J_{1}\left(\begin{array}{c}
\frac{\delta}{\delta u_{1}} \\
\frac{\delta}{\delta u_{2}}
\end{array}\right) H_{n}=J_{1} \frac{\delta H_{n}}{\delta u},
$$

where $H_{n}=-(1 / n)\left[v_{1, n+1}+\left(u_{1}-u_{2}\right) *\left(v_{2, n}+v_{3, n}\right) / 2\right]$.

Next, we will derive the Guo hierarchy with self-consistent sources. Consider the linear problem

$$
\left(\begin{array}{l}
\psi_{1, j} \\
\psi_{2, j}
\end{array}\right)_{x}=U\left(u, \lambda_{j}\right)\left(\begin{array}{l}
\psi_{1, j} \\
\psi_{2, j}
\end{array}\right), \quad\left(\begin{array}{l}
\psi_{1, j} \\
\psi_{2, j}
\end{array}\right)_{t}=V\left(u, \lambda_{j}\right)\left(\begin{array}{l}
\psi_{1, j} \\
\psi_{2, j}
\end{array}\right)
$$

The following equation was given in [21]

$$
\frac{\delta H_{k}}{\delta u}+\sum_{j=1}^{N} \alpha_{j} \frac{\delta \lambda_{j}}{\delta u}=0,
$$

where $\alpha_{j}$ are constants, and $\delta H_{k} / \delta u$ determines a finite-dimensional invariant set for the flow (2.21). Here we get [27]

$$
\begin{gathered}
\frac{\delta \lambda_{j}}{\delta u}=\frac{1}{2}\left\{\Psi_{j}, \frac{\partial U\left(u, \lambda_{j}\right)}{\partial u}\right\}, \quad \Psi_{j}=\left(\phi_{1, j} * \phi_{2, j},-\phi_{1, j} * \phi_{1, j}, \phi_{2, j} * \phi_{2, j}\right), \\
\sum_{j=1}^{N} \frac{\delta \lambda_{j}}{\delta u}=\frac{1}{4}\left(\begin{array}{c}
-\wedge\left\langle\Phi_{1}, \Phi_{1}\right\rangle+\left\langle\Phi_{2}, \Phi_{2}\right\rangle \\
\wedge\left\langle\Phi_{1}, \Phi_{1}\right\rangle+\left\langle\Phi_{2}, \Phi_{2}\right\rangle
\end{array}\right),
\end{gathered}
$$

where $\phi_{i, j}=\left(\phi_{i_{1}, j}, \phi_{i_{2}, j}, \ldots, \phi_{i_{M}, j}\right)^{T}, \wedge=\operatorname{diag}\left(\lambda_{1}, \ldots, \lambda_{N}\right), \Phi_{i}=\left(\phi_{i, 1}, \ldots, \phi_{i, N}\right)^{T}, i=1,2 ; j=$ $1, \ldots, N ;\langle\cdot, \cdot\rangle$ stands for the inner product in $R^{N}$. 
Then, according to (2.23) and taking $\alpha_{i}=-4$, the Guo hierarchy with self-consistent sources can be rewritten as follows:

$$
\left(\begin{array}{l}
u_{1} \\
u_{2}
\end{array}\right)_{t}=J_{1} \frac{\delta H_{n}}{\delta u}+J_{1}\left(\begin{array}{c}
-\wedge\left\langle\Phi_{1}, \Phi_{1}\right\rangle+\left\langle\Phi_{2}, \Phi_{2}\right\rangle \\
\wedge\left\langle\Phi_{1}, \Phi_{1}\right\rangle+\left\langle\Phi_{2}, \Phi_{2}\right\rangle
\end{array}\right)
$$

Taking $n=1$, we obtain the following multicomponent equations with self-consistent sources

$$
\begin{aligned}
& u_{1, t}-u_{2, x}-u_{1} * \frac{\left(u_{2} * u_{2}-u_{1} * u_{1}\right)}{2}=-\partial \sum_{j=1}^{N} \lambda_{j} \phi_{1 j}^{2}+\partial \sum_{j=1}^{N} \phi_{2 j}^{2}, \\
& u_{2, t}-u_{1, x}-u_{2} * \frac{\left(u_{2} * u_{2}-u_{1} * u_{1}\right)}{2}=-\partial \sum_{j=1}^{N} \lambda_{j} \phi_{1 j}^{2}-\partial \sum_{j=1}^{N} \phi_{2 j}^{2} .
\end{aligned}
$$

\section{Nonlinear Bi-Integrable Couplings of Multicomponent Guo Hierarchy with Self-Consistent Sources}

The multicomponent Guo hierarchy has been given in the above. In the section, we will construct the nonlinear bi-integrable couplings of multicomponent Guo hierarchy with selfconsistent sources by following the way in [19], which is introduced as follows.

Given an integrable equation

$$
u_{t}=K(u)
$$

which possesses a zero curvature representation

$$
U_{t}-V_{x}+[U, V]=0,
$$

where $U=U(u, \lambda), V=V(u, \lambda)$, and taking $\bar{U}=\bar{U}(\bar{u}, \lambda)=\left(U(u, \lambda), U_{1}\left(u_{1}, \lambda\right), U_{2}\left(u_{2}, \lambda\right)\right), \bar{V}=$ $\bar{V}(\bar{u}, \lambda)=\left(V(u, \lambda), V_{1}\left(u_{1}, \lambda\right), V_{2}\left(u_{2}, \lambda\right)\right)$, then the enlarged zero-curvature equation

$$
\bar{U}_{t}-\bar{V}_{x}+[\bar{U}, \bar{V}]=0
$$

equivalently yields

$$
\begin{gathered}
U_{t}-V_{x}+[U, V]=0, \\
U_{1, t}-V_{1, x}+\left[U, V_{1}\right]+\left[U_{1}, V\right]+\left[U_{1}, V_{1}\right]=0, \\
U_{2, t}-V_{2, x}+\left[U, V_{2}\right]+\left[U_{2}, V\right]+\left[U_{1}, V_{1}\right]+\left[U_{1}, V_{2}\right]+\left[U_{2}, V_{1}\right]=0 .
\end{gathered}
$$

We call (3.4) bi-integrable couplings of (3.1). Note that it is nonlinear with respect to the two supplementary variables $u_{1}, u_{2}$, thereby providing candidates for nonlinear bi-integrable couplings. 
Next, we will construct an expanding Lie algebra $Q_{M}$ of the Lie algebra $F_{M}$ and its corresponding loop algebra. Consider a vector space $Q_{M}=\operatorname{span}\left\{a=\left(a_{i j}\right)_{M \times 9}=\left(a_{1}, \ldots, a_{9}\right)\right\}$, where $a_{i}(i=1, \ldots, 9)$ is the $i$ th column of the matrix $a$.

Definition 3.1. Set $a, b$ as two elements of $Q_{M}$, their commutation operation is defined as

$$
\begin{aligned}
& {[a, b]=\left(a_{2} * b_{3}-a_{3} * b_{2}, 2\left(a_{1} * b_{2}-a_{2} * b_{1}\right), 2\left(a_{3} * b_{1}-a_{1} * b_{3}\right), a_{2} * b_{6}-a_{6} * b_{2}+a_{5} * b_{3}\right.} \\
&- a_{3} * b_{5}+a_{5} * b_{6}-a_{6} * b_{5}, 2\left(a_{1} * b_{5}-a_{5} * b_{1}+a_{4} * b_{2}-a_{2} * b_{4}+a_{4} * b_{5}-a_{5} * b_{4}\right), \\
& 2\left(a_{6} * b_{1}-a_{1} * b_{6}+a_{3} * b_{4}-a_{4} * b_{3}+a_{6} * b_{4}-a_{4} * b_{6}\right), a_{2} * b_{9}-a_{9} * b_{2}+a_{8} * b_{3} \\
&-a_{3} * b_{8}+a_{5} * b_{6}-a_{6} * b_{5}+a_{5} * b_{9}-a_{9} * b_{5}+a_{8} * b_{6}-a_{6} * b_{8}, \\
& 2\left(a_{1} * b_{8}-a_{8} * b_{1}+a_{7} * b_{2}-a_{2} * b_{7}+a_{4} * b_{5}-a_{5} * b_{4}+a_{4} * b_{8}-a_{8} * b_{4}\right. \\
&\left.+a_{7} * b_{5}-a_{5} * b_{7}\right), \\
& 2\left(a_{9} * b_{1}-a_{1} * b_{9}+a_{3} * b_{7}-a_{7} * b_{3}+a_{6} * b_{4}-a_{4} * b_{6}+a_{9} * b_{4}-a_{4} * b_{9}\right. \\
&\left.\left.+a_{6} * b_{7}-a_{7} * b_{6}\right)\right)^{T} .
\end{aligned}
$$

It is easy to verify that $Q_{M}$ consists of a Lie algebra along with (3.5). Its corresponding loop algebra $\widetilde{Q}_{M}$ is given by

$$
\widetilde{Q}_{M}=\left\{a \lambda^{n}, a \in Q_{M}, n=0, \pm 1, \pm 2, \ldots\right\}
$$

equipped with a commutation operation as follows:

$$
\left[a \lambda^{m}, b \lambda^{n}\right]=[a, b] \lambda^{m+n}, \quad \forall a, b \in Q_{M}
$$

Denoting $\widetilde{Q}_{M}(1)=\left\{\left(a_{1}, a_{2}, a_{3}, 0,0,0,0,0,0\right) \lambda^{n}\right\}$ and $\widetilde{Q}_{M}(2)=\left\{\left(0,0,0, a_{4}, a_{5}, a_{6}, a_{7}, a_{8}, a_{9}\right) \lambda^{n}\right\}$, then we have

$$
\widetilde{Q}_{M}=\widetilde{Q}_{M}(1) \oplus \widetilde{Q}_{M}(2), \quad \widetilde{Q}_{M}(1) \cong \widetilde{F}_{M}, \quad\left[\widetilde{Q}_{M}(1), \widetilde{Q}_{M}(2)\right] \subset \widetilde{Q}_{M}(2) .
$$

Here we note that the subalgebras $\widetilde{Q}_{M}(1)$ and $\widetilde{Q}_{M}(2)$ are both non-semisimple, which is very important for deriving the nonlinear integrable couplings of hierarchy.

In terms of the loop algebra $\widetilde{Q}_{M}$, consider an isospectral problem as follows:

$$
\psi_{x}=U \psi, \quad U=\frac{1}{2}\left(\lambda I_{M}, u_{1}+u_{2}, \lambda\left(u_{1}-u_{2}\right), 0, u_{3}+u_{4}, \lambda\left(u_{3}-u_{4}\right), 0, u_{5}+u_{6}, \lambda\left(u_{5}-u_{6}\right)\right)^{T} .
$$

Set

$$
V=\left(\lambda v_{1}, v_{2}+v_{3}, \lambda\left(v_{2}-v_{3}\right), \lambda v_{4}, v_{5}+v_{6}, \lambda\left(v_{5}-v_{6}\right), \lambda v_{7}, v_{8}+v_{9}, \lambda\left(v_{8}-v_{9}\right)\right)^{T}
$$


where $v_{k}=\sum_{m=0}^{\infty}\left(v_{k_{1}, m}, v_{k_{2}, m}, \ldots, v_{k_{M}, m}\right)^{T} \lambda^{-m}, k=1, \ldots, 9$. A solution to $V_{x}=[U, V]$ gives

$$
\begin{aligned}
& v_{1, m x}=u_{2} * v_{2, m}-u_{1} * v_{3, m}, \\
& v_{2, m x}=v_{3, m+1}-u_{2} * v_{1, m+1}, \\
& v_{3, m x}=v_{2, m+1}-u_{1} * v_{1, m+1}, \\
& v_{4, m x}=u_{2} * v_{5, m}-u_{1} * v_{6, m}+u_{4} * v_{2, m}-u_{3} * v_{3, m}+u_{4} * v_{5, m}-u_{3} * v_{6, m}, \\
& v_{5, m x}=2 v_{6, m+1}-u_{2} * v_{4, m+1}+v_{3, m+1}-u_{4} * v_{1, m+1}-u_{4} * v_{4, m+1}, \\
& v_{6, m x}=2 v_{5, m+1}-u_{1} * v_{4, m+1}+v_{2, m+1}-u_{3} * v_{1, m+1}-u_{3} * v_{4, m+1}, \\
& v_{7, m x}=u_{2} * v_{8, m}-u_{1} * v_{9, m}+u_{6} * v_{2, m}-u_{5} * v_{3, m}+u_{4} * v_{5, m}-u_{3} * v_{6, m} \\
& +u_{4} * v_{8, m}-u_{3} * v_{9, m}+u_{6} * v_{5, m}-u_{5} * v_{6, m}, \\
& v_{8, m x}=2 v_{9, m+1}-u_{2} * v_{7, m+1}+v_{3, m+1}-u_{6} * v_{1, m+1}+2 v_{6, m+1}-u_{4} * v_{4, m+1} \\
& -u_{4} * v_{7, m+1}-u_{6} * v_{4, m+1}, \\
& v_{9, m x}=2 v_{8, m+1}-u_{1} * v_{7, m+1}+v_{2, m+1}-u_{5} * v_{1, m+1}+2 v_{5, m+1}-u_{3} * v_{4, m+1} \\
& -u_{3} * v_{7, m+1}-u_{5} * v_{4, m+1}, \\
& v_{1,0}=0 I_{M}, \quad v_{2,0}=u_{1}, \quad v_{3,0}=u_{2}, \quad v_{4,0}=0 I_{M}, \\
& v_{5,0}=u_{1}+2 u_{3}, \quad v_{6,0}=u_{2}+2 u_{4}, \quad v_{7,0}=0 I_{M}, \\
& v_{8,0}=u_{1}+2 u_{3}+2 u_{5}, \quad v_{9,0}=u_{2}+2 u_{4}+2 u_{6}, \quad v_{1,1}=\frac{\left(u_{2} * u_{2}-u_{1} * u_{1}\right)}{2}, \\
& v_{2,1}=u_{2, x}+u_{1} * \frac{\left(u_{2} * u_{2}-u_{1} * u_{1}\right)}{2}, \quad v_{3,1}=u_{1, x}+u_{2} * \frac{\left(u_{2} * u_{2}-u_{1} * u_{1}\right)}{2}, \\
& v_{4,1}=\frac{\left[\left(u_{2}+u_{4}\right) *\left(u_{2}+u_{4}\right)-\left(u_{1}+u_{3}\right) *\left(u_{1}+u_{3}\right)\right]}{2}-\frac{\left(u_{2} * u_{2}-u_{1} * u_{1}\right)}{2}, \\
& v_{5,1}=u_{4, x}-\frac{u_{1} *\left(u_{2} * u_{2}-u_{1} * u_{1}\right)}{2}+\frac{\left(u_{1}+u_{3}\right) *\left[\left(u_{2}+u_{4}\right) *\left(u_{2}+u_{4}\right)-\left(u_{1}+u_{3}\right) *\left(u_{1}+u_{3}\right)\right]}{4}, \\
& v_{6,1}=u_{3, x}-\frac{u_{2} *\left(u_{2} * u_{2}-u_{1} * u_{1}\right)}{2}+\frac{\left(u_{2}+u_{4}\right) *\left[\left(u_{2}+u_{4}\right) *\left(u_{2}+u_{4}\right)-\left(u_{1}+u_{3}\right) *\left(u_{1}+u_{3}\right)\right]}{4}, \\
& v_{7,1}=\frac{\left(u_{4} * u_{4}-u_{3} * u_{3}\right)}{2}+\left[\left(u_{2}+u_{4}\right) * u_{6}-\left(u_{1}+u_{3}\right) * u_{5}\right] \\
& v_{8,1}=u_{6, x}+\frac{\left(u_{5}-u_{1}\right) *\left[\left(u_{2}+u_{4}\right) *\left(u_{2}+u_{4}\right)-\left(u_{1}+u_{3}\right) *\left(u_{1}+u_{3}\right)\right]}{4} \\
& +\frac{\left(u_{1}-u_{3}\right) *\left(u_{2} * u_{2}-u_{1} * u_{1}\right)}{4}+\frac{\left(u_{1}+u_{3}\right) *\left(u_{4} * u_{4}-u_{3} * u_{3}\right)}{4} \\
& +\frac{\left(u_{1}+u_{3}\right) *\left[\left(u_{2}+u_{4}\right) * u_{6}-\left(u_{1}+u_{3}\right) * u_{5}\right]}{2}, \\
& v_{9,1}=u_{5, x}+\frac{\left(u_{6}-u_{2}\right) *\left[\left(u_{2}+u_{4}\right) *\left(u_{2}+u_{4}\right)-\left(u_{1}+u_{3}\right) *\left(u_{1}+u_{3}\right)\right]}{4} \\
& +\frac{\left(u_{2}-u_{4}\right) *\left(u_{2} * u_{2}-u_{1} * u_{1}\right)}{4}+\frac{\left(u_{2}+u_{4}\right) *\left(u_{4} * u_{4}-u_{3} * u_{3}\right)}{4} \\
& +\frac{\left(u_{2}+u_{4}\right) *\left[\left(u_{2}+u_{4}\right) * u_{6}-\left(u_{1}+u_{3}\right) * u_{5}\right]}{2} .
\end{aligned}
$$


Similar to (2.9), we obtain

$$
\begin{aligned}
-V_{+x}^{(n)}+\left[U, V_{+}^{(n)}\right]=( & 0, \frac{\left(v_{2, n x}+v_{3, n x}\right)}{2}, \frac{\lambda\left(v_{2, n x}-v_{3, n x}\right)}{2}, 0, v_{5, n x}+v_{6, n x}+\frac{\left(v_{2, n x}+v_{3, n x}\right)}{2}, \\
& \lambda\left(v_{5, n x}-v_{6, n x}\right)+\frac{\lambda\left(v_{2, n x}-v_{3, n x}\right)}{2}, 0,\left(v_{8, n x}+v_{9, n x}+v_{5, n x}+v_{6, n x}\right) \\
& \left.+\frac{\left(v_{2, n x}+v_{3, n x}\right)}{2},\left(v_{8, n x}-v_{9, n x}+v_{5, n x}-v_{6, n x}\right)+\frac{\left(v_{2, n x}-v_{3, n x}\right)}{2}\right) .
\end{aligned}
$$

Therefore, the zero-curvature equation

$$
U_{t}-V_{x}^{(n)}+\left[U, V^{(n)}\right]=0
$$

admits the following bigger integrable system:

$$
\begin{aligned}
U_{t}=\left(\begin{array}{l}
u_{1} \\
u_{2} \\
u_{3} \\
u_{4} \\
u_{5} \\
u_{6}
\end{array}\right)_{t}=\left(\begin{array}{cccccc}
\partial & 0 & -\partial & 0 & \partial & 0 \\
0 & -\partial & 0 & \partial & 0 & -\partial \\
-\partial & 0 & \partial & 0 & \partial & 0 \\
0 & \partial & 0 & -\partial & 0 & -\partial \\
\partial & 0 & \partial & 0 & -\partial & 0 \\
0 & -\partial & 0 & -\partial & 0 & \partial
\end{array}\right)\left(\begin{array}{c}
v_{2, n}+v_{5, n}+v_{8, n} \\
-v_{3, n}-v_{6, n}-v_{9, n} \\
v_{2, n}+2 v_{5, n}+v_{8, n} \\
-v_{3, n}-2 v_{6, n}-v_{9, n} \\
v_{2, n}+v_{5, n} \\
-v_{3, n}-v_{6, n}
\end{array}\right) \\
\\
=J_{2} P_{n}=J_{2} L_{2}^{n}\left(\begin{array}{c}
v_{2,0}+v_{5,0}+v_{8,0} \\
-v_{3,0}-v_{6,0}-v_{9,0} \\
v_{2,0}+2 v_{5,0}+v_{8,0} \\
-v_{3,0}-2 v_{6,0}-v_{9,0} \\
v_{2,0}+v_{5,0} \\
-v_{3,0}-v_{6,0}
\end{array}\right)
\end{aligned}
$$

where $J_{2}$ is the Hamiltonian operator. Similar to (2.13), by employing (3.11), after tedious calculation, the recurrence operator $L_{2}$ can be obtained and here we omit it.

Taking $u_{3}=u_{4}=0$ in (3.14) reduces to an integrable coupling of the Guo hierarchy; taking $u_{5}=u_{6}=0$ in (3.14) reduces to another integrable coupling of the Guo hierarchy. Here we note that except the first variables $u_{1}, u_{2}$, all integrable couplings presented above are nonlinear, since the supplementary systems (3.14) with $n \geq 1$ are nonlinear with respect to the four dependent variables $u_{3}, u_{4}, u_{5}, u_{6}$. So, in term of the definition of integrable couplings, we conclude that the system (3.14) is nonlinear bi-integrable couplings of the multicomponent Guo hierarchy.

In order to deduce to the Hamiltonian structure of (3.14), we rewrite (3.5) as follows:

$$
[a, b]=a^{T} R(b),
$$


where

$R(b)$

$$
=\left(\begin{array}{ccccccccc}
0 & 2 b_{2} & -2 b_{3} & 0 & 2 b_{5} & -2 b_{6} & 0 & 2 b_{8} & -2 b_{9} \\
b_{3} & -2 b_{1} & 0 & b_{6} & -2 b_{4} & 0 & b_{9} & -2 b_{7} & 0 \\
-b_{2} & 0 & 2 b_{1} & -b_{5} & 0 & 2 b_{4} & -b_{8} & 0 & 2 b_{7} \\
0 & 0 & 0 & 0 & 2\left(b_{2}+b_{5}\right) & -2\left(b_{3}+2 b_{6}\right) & 0 & 2\left(b_{5}+b_{8}\right) & -2\left(b_{6}+b_{9}\right) \\
0 & 0 & 0 & b_{3}+b_{6} & -2\left(b_{1}+b_{4}\right) & 0 & b_{6}+b_{9} & -2\left(b_{4}+b_{7}\right) & 0 \\
0 & 0 & 0 & -b_{2}-b_{5} & 0 & 2\left(b_{1}+b_{4}\right) & -b_{5}-b_{8} & 0 & 2\left(b_{4}+b_{7}\right) \\
0 & 0 & 0 & 0 & 0 & 0 & 0 & 2\left(b_{2}+b_{5}\right) & -2\left(b_{3}+b_{6}\right) \\
0 & 0 & 0 & 0 & 0 & 0 & b_{3}+b_{6} & -2\left(b_{1}+b_{4}\right) & 0 \\
0 & 0 & 0 & 0 & 0 & 0 & -\left(b_{2}+b_{5}\right) & 0 & 2\left(b_{1}+b_{4}\right)
\end{array}\right) .
$$

Solving the matrix equation for the constant matrix $F: R(b) * F=-(R(b) * F)^{T}, F^{T}=F$, presents

$$
F=\left(\begin{array}{ccccccccc}
2 I_{M} & 0 & 0 & 2 I_{M} & 0 & 0 & 2 I_{M} & 0 & 0 \\
0 & 0 & I_{M} & 0 & 0 & I_{M} & 0 & 0 & I_{M} \\
0 & I_{M} & 0 & 0 & I_{M} & 0 & 0 & I_{M} & 0 \\
2 I_{M} & 0 & 0 & 4 I_{M} & 0 & 0 & 2 I_{M} & 0 & 0 \\
0 & 0 & I_{M} & 0 & 0 & 2 I_{M} & 0 & 0 & I_{M} \\
0 & I_{M} & 0 & 0 & 2 I_{M} & 0 & 0 & I_{M} & 0 \\
2 I_{M} & 0 & 0 & 2 I_{M} & 0 & 0 & 0 & 0 & 0 \\
0 & 0 & I_{M} & 0 & 0 & I_{M} & 0 & 0 & 0 \\
0 & I_{M} & 0 & 0 & I_{M} & 0 & 0 & 0 & 0
\end{array}\right) .
$$

Then in terms of $F$, define a linear functional

$$
\begin{aligned}
\{a, b\}= & a^{T} * F * b \\
= & 2\left(a_{1}+a_{4}+a_{7}\right) * b_{1}+\left(a_{3}+a_{6}+a_{9}\right) * b_{2} \\
& +\left(a_{2}+a_{5}+a_{8}\right) * b_{3}+2\left(a_{1}+2 a_{4}+a_{7}\right) * b_{4}+\left(a_{3}+2 a_{6}+a_{9}\right) * b_{5} \\
& +\left(a_{2}+2 a_{5}+a_{8}\right) * b_{6}+2\left(a_{1}+a_{4}\right) * b_{7}+\left(a_{3}+a_{6}\right) * b_{8}+\left(a_{2}+a_{5}\right) * b_{9} .
\end{aligned}
$$

It is easy to find that $\{a, b\}$ satisfies the variational identity (2.18). By using the above linear functional, we have

$$
\begin{aligned}
\left\{V, U_{\lambda}\right\}= & \lambda\left(v_{1}+v_{4}+v_{7}\right)+\frac{1}{2}\left(v_{2}+v_{3}+v_{5}+v_{6}+v_{8}+v_{9}\right) *\left(u_{1}-u_{2}\right) \\
& +\frac{1}{2}\left(v_{2}+v_{3}+2 v_{5}+2 v_{6}+v_{8}+v_{9}\right) *\left(u_{3}-u_{4}\right)+\frac{1}{2}\left(v_{2}+v_{3}+v_{5}+v_{6}\right) *\left(u_{5}-u_{6}\right), \\
\left\{V, U_{u_{1}}\right\}= & \lambda\left(v_{2}+v_{5}+v_{8}\right), \quad\left\{V, U_{u_{2}}\right\}=\lambda\left(-v_{3}-v_{6}-v_{9}\right), \quad\left\{V, U_{u_{3}}\right\}=\lambda\left(v_{2}+2 v_{5}+v_{8}\right), \\
\left\{V, U_{u_{4}}\right\}= & \lambda\left(-v_{3}-v_{6}-v_{9}\right), \quad\left\{V, U_{u_{5}}\right\}=\lambda\left(v_{2}+v_{5}\right), \quad\left\{V, U_{u_{6}}\right\}=\lambda\left(-v_{3}-v_{6}\right) .
\end{aligned}
$$


Substituting (3.19) into the variational identity (2.18), we have

$$
\begin{gathered}
\frac{\delta}{\delta u}\left(\lambda\left(v_{1}+v_{4}+v_{7}\right)+\frac{1}{2}\left(v_{2}+v_{3}+v_{5}+v_{6}+v_{8}+v_{9}\right) *\left(u_{1}-u_{2}\right)\right. \\
\left.+\frac{1}{2}\left(v_{2}+v_{3}+2 v_{5}+2 v_{6}+v_{8}+v_{9}\right) *\left(u_{3}-u_{4}\right)+\frac{1}{2}\left(v_{2}+v_{3}+v_{5}+v_{6}\right) *\left(u_{5}-u_{6}\right)\right) \\
=\lambda^{-r} \frac{\partial}{\partial \lambda} \lambda^{r}\left(\lambda\left(v_{2}+v_{5}+v_{8}\right), \lambda\left(-v_{3}-v_{6}-v_{9}\right), \lambda\left(v_{2}+2 v_{5}+v_{8}\right), \lambda\left(-v_{3}-v_{6}-v_{9}\right),\right. \\
\left.\lambda\left(v_{2}+v_{5}\right), \lambda\left(-v_{3}-v_{6}\right)\right)^{T} .
\end{gathered}
$$

Comparing the coefficients of $\lambda^{-n-1}$ yields

$$
\begin{aligned}
\frac{\delta}{\delta u}\left(v_{1, n+1}+v_{4, n+1}+v_{7, n+1}+\frac{1}{2}\left(v_{2, n}+v_{3, n}+v_{5, n}+v_{6, n}+v_{8, n}+v_{9, n}\right) *\left(u_{1}-u_{2}\right)\right. \\
+\frac{1}{2}\left(v_{2, n}+v_{3, n}+2 v_{5, n}+2 v_{6, n}+v_{8, n}+v_{9, n}\right)\left(u_{3}-u_{4}\right) \\
\left.+\frac{1}{2}\left(v_{2, n}+v_{3, n}+v_{5, n}+v_{6, n}\right) *\left(u_{5}-u_{6}\right)\right) \\
=(\gamma-n)\left(\left(v_{2, n}+v_{5, n}+v_{8, n}\right),\left(-v_{3, n}-v_{6, n}-v_{9, n}\right),\left(v_{2, n}+2 v_{5, n}+v_{8, n}\right),\right. \\
\left.\left(-v_{3, n}-2 v_{6, n}-v_{9, n}\right),\left(v_{2, n}+v_{5, n}\right),\left(-v_{3, n}-v_{6, n}\right)\right)^{T} .
\end{aligned}
$$

Taking $n=0$ in the above equation gives $\gamma=0$.

Hence, the nonlinear bi-integrable couplings of the multicomponent Guo hierarchy (3.14) can be written as a Hamiltonian form

$$
U_{t}=\left(\begin{array}{l}
u_{1} \\
u_{2} \\
u_{3} \\
u_{4} \\
u_{5} \\
u_{6}
\end{array}\right)_{t}=J_{2}\left(\begin{array}{c}
\frac{\delta}{\delta u_{1}} \\
\frac{\delta}{\delta u_{2}} \\
\frac{\delta}{\delta u_{3}} \\
\frac{\delta}{\delta u_{4}} \\
\frac{\delta}{\delta u_{5}} \\
\frac{\delta}{\delta u_{6}}
\end{array}\right) \bar{H}_{n}=J_{2} \frac{\delta \bar{H}_{n}}{\delta u},
$$

where $\bar{H}_{n}=-(1 / n)\left[v_{1, n+1}+v_{4, n+1}+v_{7, n+1}+(1 / 2)\left(v_{2, n}+v_{3, n}+v_{5, n}+v_{6, n}+v_{8, n}+v_{9, n}\right) *\left(u_{1}-u_{2}\right)+\right.$ $\left.(1 / 2)\left(v_{2, n}+v_{3, n}+2 v_{5, n}+2 v_{6, n}+v_{8, n}+v_{9, n}\right)\left(u_{3}-u_{4}\right)+(1 / 2)\left(v_{2, n}+v_{3, n}+v_{5, n}+v_{6, n}\right) *\left(u_{5}-u_{6}\right)\right]$. 
Next, we will derive the nonlinear bi-integrable couplings of the multicomponent Guo hierarchy with self-consistent sources. Consider the linear problem

$$
\left(\begin{array}{l}
\psi_{1, j} \\
\psi_{2, j} \\
\psi_{3, j} \\
\psi_{4, j} \\
\psi_{5, j} \\
\psi_{6, j}
\end{array}\right)_{x}=U\left(u, \lambda_{j}\right)\left(\begin{array}{l}
\psi_{1, j} \\
\psi_{2, j} \\
\psi_{3, j} \\
\psi_{4, j} \\
\psi_{2, j} \\
\psi_{6, j}
\end{array}\right), \quad\left(\begin{array}{l}
\psi_{1, j} \\
\psi_{2, j} \\
\psi_{3, j} \\
\psi_{4, j} \\
\psi_{5, j} \\
\psi_{6, j}
\end{array}\right)_{t}=V\left(u, \lambda_{j}\right)\left(\begin{array}{l}
\psi_{1, j} \\
\psi_{2, j} \\
\psi_{3, j} \\
\psi_{4, j} \\
\psi_{5, j} \\
\psi_{6, j}
\end{array}\right) .
$$

According to (2.24) and the definition of $\{a, b\}$, we get

$$
\begin{aligned}
\Psi_{j}=( & \phi_{1, j} * \phi_{2, j},-\phi_{1, j} * \phi_{1, j}, \phi_{2, j} * \phi_{2, j}, \phi_{3, j} * \phi_{4, j},-\phi_{3, j} * \phi_{3, j}, \phi_{4, j} * \phi_{4, j}, \phi_{5, j} * \phi_{6, j}, \\
& \left.-\phi_{5, j} * \phi_{5, j}, \phi_{6, j} * \phi_{6, j}\right), \\
\sum_{j=1}^{N} \frac{\delta \lambda_{j}}{\delta u}= & \frac{1}{4} W \\
= & \frac{1}{4}\left(\begin{array}{c}
\left\langle\Phi_{2}, \Phi_{2}\right\rangle+\left\langle\Phi_{4}, \Phi_{4}\right\rangle+\left\langle\Phi_{6}, \Phi_{6}\right\rangle-\wedge\left(\left\langle\Phi_{1}, \Phi_{1}\right\rangle+\left\langle\Phi_{3}, \Phi_{3}\right\rangle+\left\langle\Phi_{5}, \Phi_{5}\right\rangle\right) \\
\left\langle\Phi_{2}, \Phi_{2}\right\rangle+2\left\langle\Phi_{4}, \Phi_{4}\right\rangle+\left\langle\Phi_{6}, \Phi_{6}\right\rangle+\left\langle\Phi_{6}, \Phi_{6}\right\rangle-\wedge\left(\left\langle\Phi_{1}, \Phi_{1}\right\rangle+\left\langle\Phi_{1}, \Phi_{1}\right\rangle+2\left\langle\Phi_{3}\right\rangle+\left\langle\Phi_{5}, \Phi_{3}\right\rangle+\left\langle\Phi_{5}\right\rangle\right) \\
\left.\left\langle\Phi_{2}, \Phi_{2}\right\rangle+2\left\langle\Phi_{4}\right\rangle\right) \\
\left\langle\Phi_{2}, \Phi_{4}\right\rangle+\left\langle\Phi_{2}\right\rangle+\left\langle\Phi_{6}, \Phi_{6}\right\rangle+\wedge\left(\left\langle\Phi_{1}, \Phi_{1}\right\rangle+2\left\langle\Phi_{3}, \Phi_{3}\right\rangle+\left\langle\Phi_{5}, \Phi_{5}\right\rangle\right) \\
\left\langle\Phi_{2}, \Phi_{2}\right\rangle+\left\langle\Phi_{1}, \Phi_{1}\right\rangle+\left\langle\Phi_{3}, \Phi_{4}\right\rangle-\wedge\left(\left\langle\Phi_{1}, \Phi_{1}\right\rangle+\left\langle\Phi_{3}, \Phi_{3}\right\rangle\right)
\end{array}\right),
\end{aligned}
$$

where $\phi_{i, j}=\left(\phi_{i_{1}, j}, \phi_{i_{2}, j}, \ldots, \phi_{i_{M}, j}\right)^{T}, \wedge=\operatorname{diag}\left(\lambda_{1}, \ldots, \lambda_{N}\right), \Phi_{i}=\left(\phi_{i, 1}, \ldots, \phi_{i, N}\right)^{T}, i=1, \ldots, 6$; $j=1, \ldots, N ;\langle\cdot, \cdot\rangle$ stands for the inner product in $R^{N}$.

Then, according to (2.23) and taking $\alpha_{i}=-4$, the nonlinear bi-integrable couplings of the multicomponent Guo hierarchy with self-consistent sources can be rewritten as follows:

$$
\left(u_{1}, u_{2}, u_{3}, u_{4}, u_{5}, u_{6}\right)_{t}^{T}=J_{2} \frac{\delta \bar{H}_{n}}{\delta u}+J_{2} W
$$

\section{Conclusions}

In this paper, we derive the multicomponent Guo hierarchy with self-consistent sources and its nonlinear bi-integrable couplings by using the Lie algebra $Q_{M}$. As far as we know, the existing papers are not designed for constructing the nonlinear bi-integrable couplings of multicomponent hierarchy with self-consistent sources. This method can be used to derive the other multicomponent soliton hierarchies with self-consistent sources and their nonlinear bi-integrable couplings. 


\section{Acknowledgments}

This work was supported by Innovation Project of Chinese Academy of Sciences (no. KZCX2EW-209) and National Natural Science Foundation of China (no. 11271007).

\section{References}

[1] W. X. Ma and B. Fuchssteiner, "Integrable theory of the perturbation equations," Chaos, Solitons and Fractals, vol. 7, no. 8, pp. 1227-1250, 1996.

[2] W. X. Ma, "Integrable couplings of soliton equations by perturbations. I: a general theory and application to the KdV hierarchy," Methods and Applications of Analysis, vol. 7, no. 1, pp. 21-55, 2000.

[3] G. Z. Tu, "The trace identity, a powerful tool for constructing the Hamiltonian structure of integrable systems," Journal of Mathematical Physics, vol. 30, no. 2, pp. 330-338, 1989.

[4] W. X. Ma, "A new hierarchy of Liouville integrable generalized Hamiltonian equations and its reduction," Chinese Journal of Contemporary Mathematics, vol. 13, no. 1, pp. 79-89, 1992.

[5] F. K. Guo, "Two hierarchies of integrable Hamiltonian equations," Mathematica Applicata, vol. 9, no. 4, pp. 495-499, 1996.

[6] Y. F. Zhang, "Two types of new Lie algebras and corresponding hierarchies of evolution equations," Physics Letters A, vol. 310, no. 1, pp. 19-24, 2003.

[7] F. K. Guo and Y. F. Zhang, "A new loop algebra and a corresponding integrable hierarchy, as well as its integrable coupling," Journal of Mathematical Physics, vol. 44, no. 12, pp. 5793-5803, 2003.

[8] W. X. Ma, "A Hamiltonian structure associated with a matrix spectral problem of arbitrary-order," Physics Letters A, vol. 367, no. 6, pp. 473-477, 2007.

[9] T. C. Xia, F. C. You, and W. Y. Zhao, "Multi-component Levi hierarchy and its multi-component integrable coupling system," Communications in Theoretical Physics, vol. 44, no. 6, pp. 990-996, 2005.

[10] H.-H. Dong and N. Zhang, "The quadratic-form identity for constructing Hamiltonian structures of the Guo hierarchy," Chinese Physics, vol. 15, no. 9, pp. 1919-1926, 2006.

[11] T. Xia, F. Yu, and Y. Zhang, "The multi-component coupled Burgers hierarchy of soliton equations and its multi-component integrable couplings system," Physica A, vol. 343, no. 15, pp. 238-246, 2004.

[12] Z. Li and H. Dong, "Integrable couplings of the multi-component dirac hierarchy and its Hamiltonian structure," Chaos, Solitons E Fractals, vol. 36, no. 2, pp. 290-295, 2008.

[13] W. X. Ma and Y. Zhang, "Component-trace identities for Hamiltonian structures," Applicable Analysis, vol. 89, no. 4, pp. 457-472, 2010.

[14] W. X. Ma, X. X. Xu, and Y. F. Zhang, "Semi-direct sums of Lie algebras and continuous integrable couplings," Physics Letters A, vol. 351, no. 3, pp. 125-130, 2006.

[15] W. X. Ma, "Variational identities and Hamiltonian structures," AIP Conference Proceedings, vol. 1212, no. 1 , pp. 1-27, 2010.

[16] W. X. Ma, "Nonlinear continuous integrable Hamiltonian couplings," Applied Mathematics and Computation, vol. 217, no. 17, pp. 7238-7244, 2011.

[17] Y. F. Zhang, "Lie algebras for constructing nonlinear integrable couplings," Communications in Theoretical Physics, vol. 56, no. 5, pp. 805-812, 2011.

[18] Y. F. Zhang and B. L. Feng, "A few Lie algebras and their applications for generating integrable hierarchies of evolution types," Communications in Nonlinear Science and Numerical Simulation, vol. 16, no. 8, pp. 3045-3061, 2011.

[19] W. X. Ma, "Loop algebras and bi-integrable couplings," Chinese Annals of Mathematics B, vol. 33, no. 2, pp. 207-224, 2012.

[20] M. Antonowicz, "Gelfand-Dikii hierarchies with the sources and Lax representation for restricted flows," Physics Letters A, vol. 165, no. 1, pp. 47-52, 1992.

[21] Y. B. Zeng, W. X. Ma, and R. Lin, "Integration of the soliton hierarchy with self-consistent sources," Journal of Mathematical Physics, vol. 41, no. 8, pp. 5453-5489, 2000.

[22] V. K. Mel'nikov, "New method for deriving nonlinear integrable systems," Journal of Mathematical Physics, vol. 31, no. 5, pp. 1106-1113, 1990.

[23] C. Claude, A. Latifi, and J. Leon, "Nonlinear resonant scattering and plasma instability: an integrable model," Journal of Mathematical Physics, vol. 32, no. 12, pp. 3310-3321, 1991.

[24] F. J. Yu, "A kind of integrable couplings of soliton equations hierarchy with self-consistent sources associated with $\tilde{s l}(4), "$ Physics Letters A, vol. 372, no. 44, pp. 6613-6621, 2008. 
[25] T. C. Xia, "Two new integrable couplings of the soliton hierarchies with self-consistent sources," Chinese Physics B, vol. 19, no. 10, Article ID 100303, 2010.

[26] F. J. Yu and L. Li, "Integrable coupling system of JM equations hierarchy with self-consistent sources," Communications in Theoretical Physics, vol. 53, no. 1, pp. 6-12, 2010.

[27] G. Z. Tu, "An extension of a theorem on gradients of conserved densities of integrable systems," Northeastern Mathematical Journal, vol. 6, no. 1, pp. 26-32, 1990. 


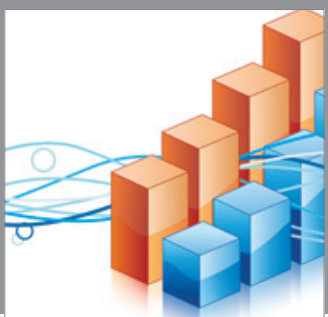

Advances in

Operations Research

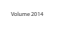

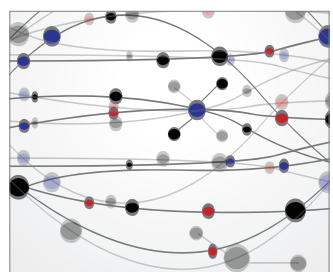

\section{The Scientific} World Journal
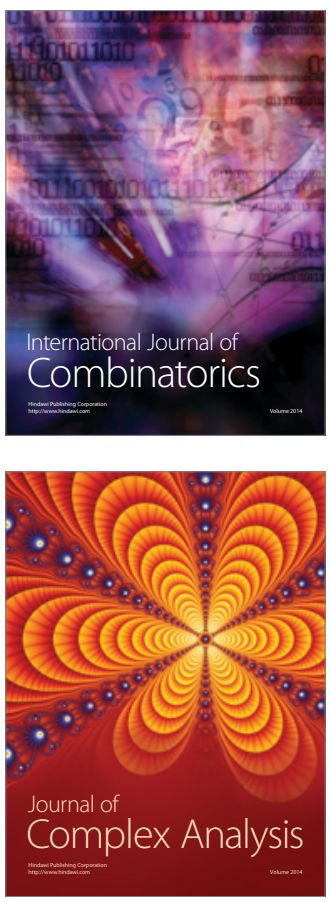

International Journal of

Mathematics and

Mathematical

Sciences
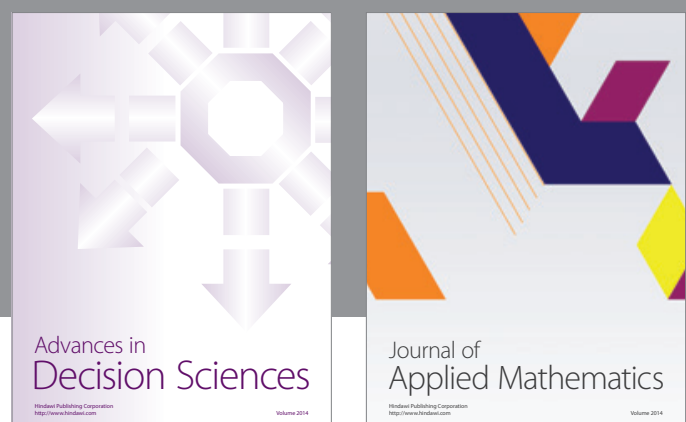

Journal of

Applied Mathematics
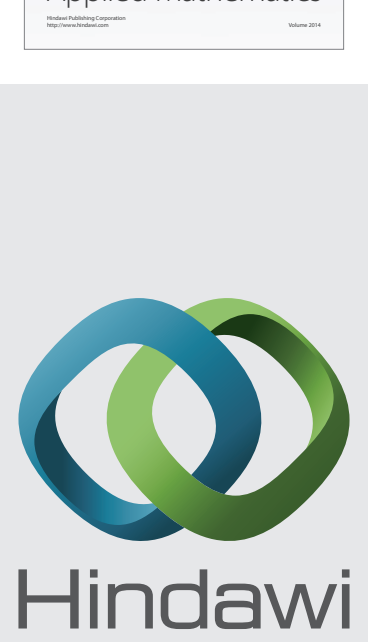

Submit your manuscripts at http://www.hindawi.com
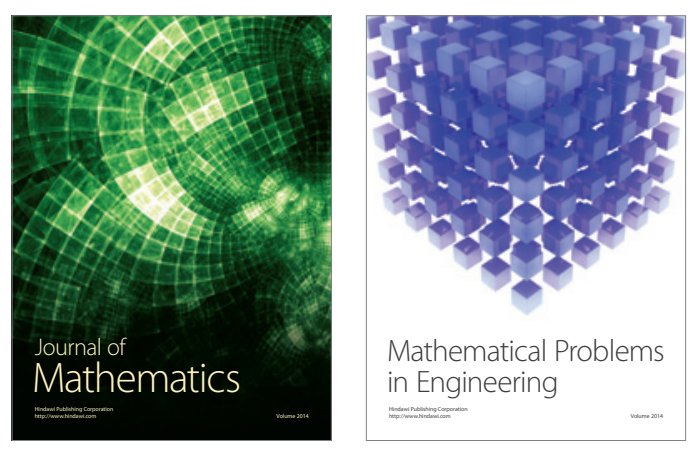

Mathematical Problems in Engineering
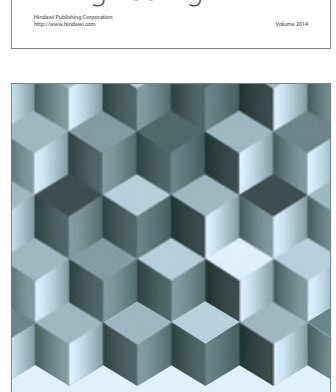

Journal of

Function Spaces
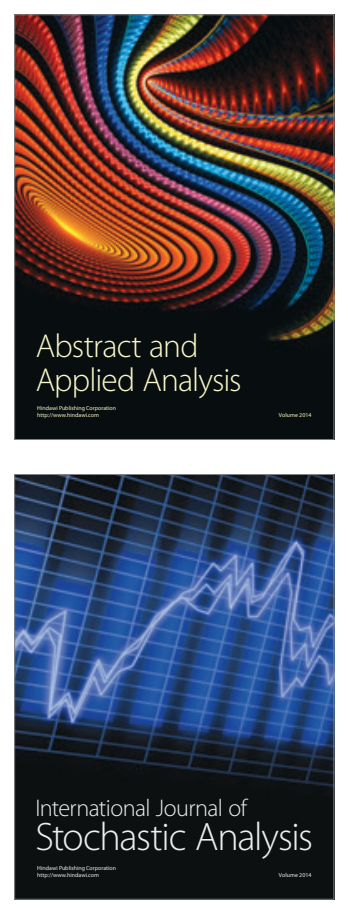

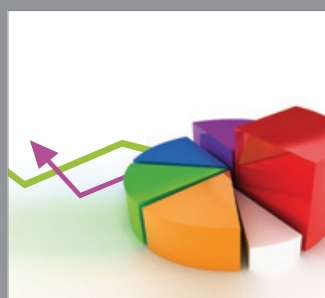

ournal of

Probability and Statistics

Promensencen
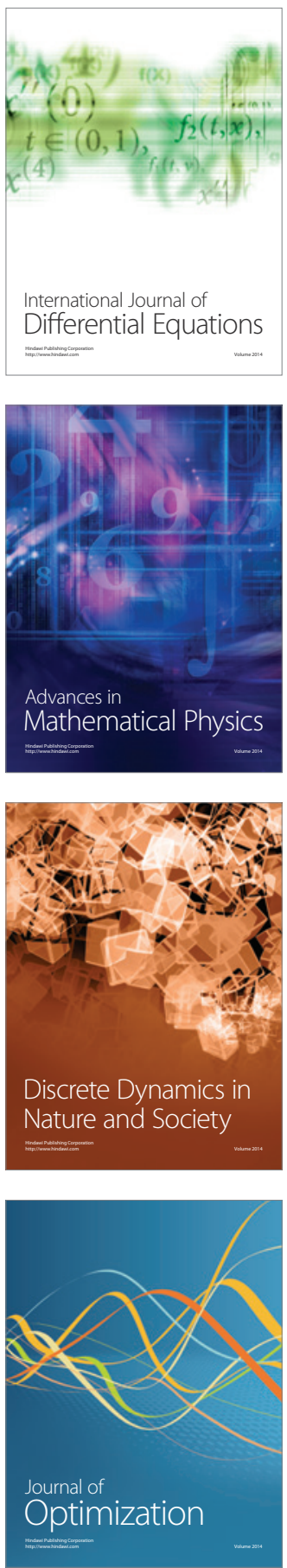